\title{
A Dual Beam Experiment for simultaneous irradiation of surfaces with ion species of gaseous and solid-state elements
}

\author{
I. Bizyukov ${ }^{(a, b)}$, K. Krieger ${ }^{(b)}$ \\ ${ }^{(a)}$ Home University: Kharkiv National University, Faculty of Physics and Technologies, \\ 31 Kurchatov Ave., Kharkiv 61108, Ukraine

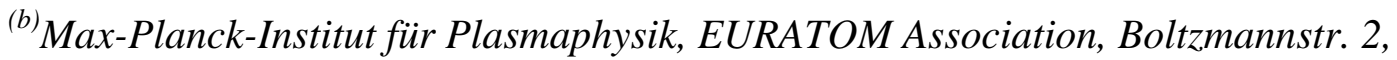 \\ .85748 Garching, Germany
}

\begin{abstract}
The effects occurring under simultaneous bombardment of surfaces by ions of gaseous and solid elements is a new field for research, which is of great interest for applications ranging from cold plasma technologies to nuclear fusion in magnetically confined plasmas. A multitude of new effects has been observed originating from mixing of incident ion species and bulk material at the surface and, in some cases, from additional complex chemical interactions between the mixing species. To study these effects under controlled conditions a new Dual Beam Experiment (DBE) has been commissioned, particularly suited for in-situ studies of surface sputtering and ion implantation processes. Thin films with negligible impurity level and surface roughness are used as targets. High energy ion beam analysis is used as a means of non-destructive elemental depth profiling and concentration measurements. This allows for the first time the fast quantification of the elemental composition change of a target sample in intervals between ion irradiation. The paper describes the details of design and operation, as well as the accessible range of experimental conditions.
\end{abstract}




\section{Introduction}

The basic physical processes of sputtering by impact of ions or atoms have been extensively investigated [1] and there is a good understanding of the theoretical modelling of these processes [2]. However, sputtering processes where the incident species is a nonvolatile element, sputtering by a mixture of volatile and non-volatile species and/or sputtering processes in the presence of diffusion due to elevated target temperature are still an open area of research, which has become particularly important for applications in nuclear fusion research, plasma processing and plasma technology. The energy range of incident particles, which is of interest for these applications ranges from typical surface binding energies of the order of a few electron Volts up to $\approx 100 \mathrm{keV}$. Apart from kinetic effects as ion scattering and diffusion in the solid, the dynamics of the sputtering processes can be strongly affected by additional processes like formation of chemical bonds or alloy formation and geometric effects such as surface morphology. Furthermore, bombardment by non-volatile elements leads to modification of the target material composition and a corresponding dependency of the target properties on the fluence of the incident species. If time scales of diffusion processes or chemical reactions are similar to those of changes of the elemental composition of the target the target properties will generally depend on the flux of the incident species.

With simultaneous bombardment by ions of volatile and non-volatile elements the sputtering processes and target modification will depend on the ratio of the incident species and will for example lead to a competition between surface erosion and implantation or layer deposition [3]. In this respect the balance point will be of particular importance, since it separates two qualitatively different processes. The physics model for the description of the surface erosion becomes more complicate because the properties of the mixed materials 
will be generally different from the original target material. At higher temperatures the sputtering dynamics may become even more complicated by diffusion of the incident species into the bulk of the target material [4] and by various chemical interactions between the species involved [5].

Sputtering by simultaneous incidence of volatile and non-volatile species has become particularly important for the field of nuclear fusion with magnetically confined plasmas. In a fusion device, the plasma facing vessel components are subject to a bombardment with the fuel species deuterium and tritium with energies from $\approx 1 \mathrm{eV}-100 \mathrm{keV}$ and energy depending fluxes up to $10^{24} \mathrm{~m}^{-2} \mathrm{~s}^{-1}$. The impact of the fuel species leads to release of wall material by sputtering, which may subsequently penetrate the fusion plasma and migrate to other locations. Upon re-deposition the incident impurity species additionally contribute to the wall sputtering and may cause modifications of the surface composition and morphology. Because of the wide parameter range of the incident particles, all major design studies of future fusion research and reactor devices employ several different plasma facing materials depending on the vessel location [6]. For regions subject to high particle fluxes such as divertor and baffle structures tungsten is envisaged as plasma-facing component (see, e.g. [7, 8, 9]). Results from the ASDEX-Upgrade tungsten-divertor experiment show that the erosion of tungsten is dominated by impact of impurity ions where $\mathrm{C}, \mathrm{W}$ and $\mathrm{O}$ are the most common species [10]. The simultaneous bombardment of the W surface by hydrogen isotopes and impurities, namely carbon and tungsten, leads to synergistic effects with significantly different ion-surface interaction properties compared to the ones of pure hydrogen or pure impurity bombardment [11].

Laboratory experiments on simultaneous bombardment of high-Z materials with hydrogen isotopes and carbon projectiles have been reported previously $[12,13]$. The simplest way to produce a combined hydrogen/carbon beam is a discharge in methane and 
irradiation of surfaces with $\mathrm{CH}_{3}$ radicals, which restricts, however, the ratio of carbon to hydrogen to 1:3. In addition the energies of both species will depend on each other. The experimental results, particularly regarding erosion yields, cannot be explained by the superposition of processes resulting from mutually independent irradiation of tungsten with carbon and hydrogen [12]. The understanding of the processes involved was further developed in [13]. One observes that pure carbon bombardment leads to continuous tungsten erosion only at elevated temperatures where diffusion causes continuous depletion of carbon from the surface. In the presence of hydrogen, this effect starts already at much lower temperature. Experimental data on erosion have been used to establish a theoretical model describing this phenomenon for the case of pure carbon bombardment. To extend the modeling to the case of simultaneous impact of carbon and hydrogen, parametric studies of the respective erosion processes are essential because of the additional chemical erosion of implanted carbon by the simultaneous impact of hydrogen, which is in the present model only included in a phenomenological description.

To study these synergistic effects, which have a great impact on the sputtering dynamics as well as on the surface conditions, a new Dual Beam Experiment (DBE) has been designed and implemented at IPP Garching, which allows a much wider parameter range and element selection than accessible in previous experiments. As an improvement over existing comparable devices [14], it allows for the first time in-situ ion beam analysis of irradiated samples. While in the previous experiments erosion and implantation processes were quantified by measuring the weight change of the sample, the new setup allows to track the evolution of the sample composition during the whole irradiation process. While weight change provides only integrated information, in-situ ion beam analysis yields detailed information on the depth distribution of the element species in the investigated sample. This paper describes the design and the accessible range of 
experimental conditions provided by the DBE setup. The experiments with carbon/deuterium irradiation of tungsten in continuation of previous studies with pure carbon beams and with $\mathrm{CH}_{3}$ beams are presented as an example for the demonstration of the DBE capabilities.

\section{Design of the DBE setup}

A schematic view of the experimental setup is shown in Figure 1. The simultaneous bombardment of the target with two different ion species is implemented by directing two respective ion beams with energies in the range of $0.5-10 \mathrm{keV}$ to the same spot on the surface. Additionally, a MeV analysis beam is directed at the central part of the irradiation spot and provides the possibility of in-situ ion beam analysis (IBA) using standard techniques like Rutherford backscattering (RBS), nuclear reaction analysis (NRA) and proton induced x-ray emission analysis (PIXE), for surface investigation.

The DBE setup includes the two ion-beam systems, the high energy beam line and a vacuum chamber. A duoplasmatron ion source provides ions of various gaseous species excluding chemically reactive species. A sputter ion-beam system provides ion beams of solid-state elements (impurity ion beams) and ions of gaseous elements sputtered from respective solid state compounds. The high energy beam line is used for delivery of the analysing high energy ion beam from the IPP tandem accelerator to the target surface. The vacuum chamber contains the irradiated target fixed on movable heating holder and equipment for IBA and diagnostic of low energy beams. Each subsystem of the setup can be pumped independently and can be separated from the target chamber by shutters.

\subsection{MeV beam line for ion beam analysis}

The high energy ion beam line connects the target chamber with the IPP $3 \mathrm{MV}$ tandem accelerator, which provides ion species for different types of ion beam analysis. 
The beam line is pumped independently by a turbo-molecular pump providing a base pressure $<10^{-8} \mathrm{mbar}$ and an operating pressure of $\approx 10^{-7} \mathrm{mbar}$. It can be sealed by two shutters when IBA is not required. Quadrupole magnet systems and a beam profile monitor are used for fine adjustment of the high energy ion beam trajectory. The high energy ion beam shares the beam defining aperture system in the target chamber with the low energy ion beam from the duoplasmatron ion-beam system. The high energy ion beam is passed into the vacuum chamber through the duoplasmatron's 60-degree analysis magnet, which is switched off during IBA measurements. In this operation mode the non-deflected low energy ion beam is passing the magnet chamber into a Faraday cup serving as beam dump area, which allows also to measure the undeflected beam current.

\subsection{Duoplasmatron ion-beam system}

The duoplasmatron ion beam system consists of standard components manufactured by Peabody Scientific (model PS-100 duoplasmatron ion source, model 300 extraction gap, model 370 Einzel lens). A customised drift tube with included electrostatic $x-y$ steerer plates is used to adapt the system to a double focusing 0.5 Tesla 60 -degree bending magnet with inclined pole shoes $\left(2.3^{\circ}\right)$ and a curvature radius of $92 \mathrm{~mm}$. The resulting focal length of the magnet is $430 \mathrm{~mm}$. With a $3 \mathrm{~mm}$ aperture at the focal point the magnet provides a mass $\times$ energy resolution of $7.4 \times 10^{-3}$. The duoplasmatron is capable of producing hydrogen isotope and noble gas ion beams with energies varying from 0.5 to $10 \mathrm{keV}$. The heated up filament in the source is made of platinum gauze, and a barium carbonate solution is used to maximise electron emission. Ions are formed and accelerated in the ion source and then extracted by the extraction gap providing a beam with an energy spread $<25 \mathrm{eV}$. The aperture size of $3 \mathrm{~mm}$ and the energy resolution of the magnet impose a lower limit of $3.4 \mathrm{keV}$ on the beam energy for $100 \%$ transmission. Below that energy a part of the beam will be cut off by the aperture due to the energy spread. The final beam focusing 
on the target position is realized by the combination of Einzel lens and 60-degree bending magnet. Fine positioning of the beam is performed by the steerer plate assembly. It consists of two orthogonal pairs of electrostatic deflection plates mounted in sequence to eliminate quadrupole focusing effects. The pressure in the discharge chamber of the duoplasmatron is a critical parameter for the optimisation of the beam current. It is stabilised at a constant adjustable level by a feedback controlled thermovalve with an analog controller using the pressure at the duoplasmatron pump duct as feedback signal.

\subsection{Sputter ion-beam system}

The sputter ion-beam system is based on a cesium sputter source, model PS-120 from Peabody Scientific. It is capable of providing a wide variety of negative ions with energies from 0.5 to $15 \mathrm{keV}$. Negatively charged ions are formed in the ion source by sputtering of a target by cesium ions. While for solid state elements, the pure material is used for the cathode, ions beams of gaseous elements can be produced by using suitable chemical compounds in solid form (hydrides, oxides). The negative ions are accelerated to ground potential and emerge with an energy equal to the cathode voltage and an energy spread $<20 \mathrm{eV}$ and are then mass analysed by a 30-degree magnet. The mass $\times$ energy resolution of the magnet is $\approx 0.03$. With a beam defining aperture of $3 \mathrm{~mm}$ this allows separation of high-Z elements from their compounds with impurities (e.g. W, mass 184 from WC, mass 192) or separation of carbon (mass 12) from $\mathrm{CH}$ (mass 13). The mass resolution is, however, sufficiently low let most isotopes of a high-Z element as tungsten pass to achieve maximum beam currents. Its maximal magnetic flux density is 0.88 Tesla and its radius of curvature is $25.4 \mathrm{~cm}$. The Einzel lens and steerer plates have the same standard design as in the case of the duoplasmatron ion-beam system. Because of the principle of operation [15], the system has a time variable beam current. The system allows to obtain sufficiently high beam currents for approximately 10 hours operation. 


\subsection{Target Chamber}

The vacuum chamber with a diameter of $350 \mathrm{~mm}$ is equipped with a flange, which provides the base for the target holder assembly, the beam guiding systems and the detector assembly. The residual pressure in the vacuum chamber during IBA measurements is $<10^{-7}$ mbar and during low energy ion irradiation increases to values $<2 \times 10^{-6}$ mbar. Partial pressure of water is presently reduced by a liquid nitrogen trap, which will be replaced by a water cryo-pump with a pumping speed of $12000 \mathrm{l} / \mathrm{s}$ to decrease the base pressure during low energy irradiation and to speed up the pumping time after target exchange. The experimental setup on the flange is shown in Figure 2. The cross-section of the beams and their position at the target is defined by beam aperture systems (1) and (3) ensuring an exact match of both beams on the irradiated surface. The beam entry aperture (1) is split in 4 electrically insulated sectors, which allows to detect beam deviations occurring in the beam line between beam profile monitor and chamber entry. To avoid edge effects on IBA measurements beam tube (1) also includes a movable exit aperture, which allows to reduce the aperture diameter of $3 \mathrm{~mm}$ used for low energy irradiation to a diameter of $1 \mathrm{~mm}$ for ion beam analysis. Consequently, only the uniformly irradiated region of the target is analysed and therefore only depth variations of the elemental concentrations have to be considered in contrast to weight loss measurements where lateral variations of the irradiation current density may lead to significant errors in the interpretation of the results.

Shutters (2) and (4) can cut off the beams independently to allow separate measurement of the beam currents. A Faraday cup (5) surrounding the target assembly suppresses secondary electrons, originating from the ion bombardment of the target. The target assembly contains two sample holders (6) and can be independently rotated horizontally and moved vertically. The target surfaces are positioned to exactly match the 
vertical rotation axis.

All detector systems for ion beam analysis are mounted on a common base plate. For RBS PIPS detectors are installed at scattering angles of $165^{\circ}(7), 135^{\circ}(8)$ and $105^{\circ}(9)$. In addition, the system is equipped with an Amptek XR-100CR Si-PIN X-ray detector with an energy resolution of $149 \mathrm{eV}$ for PIXE analysis (10) and a PIPS detector with a $12 \mu \mathrm{m}$ Mylar foil for detection of protons from nuclear reactions (11). During irradiation the detector systems are protected by a shutter (12) from backscattered ions.

The vertical cross-section of the Faraday cup shows the target holder assembly, which can be rotated and moved vertically by a two-axis manipulator system. The target holder assembly consists of a base plate with separated calibration target holder (13) and a target holder with two heaters $(14,15)$ for the investigated samples. The boron-nitride ceramic heaters (16) allow to heat the bombarded samples up to $1200^{\circ} \mathrm{C}$. To prevent excessive heating of the base plate and the adjacent calibration targets and beam viewing system, radiation shields are installed both at the backside of the heaters and between the three subsystems of the target holder assembly. A two-wavelength pyrometer is used for contactless acquisition of the sample surface temperature. The system allows measurement of temperatures $>300^{\circ} \mathrm{C}$ with a resolution of about $10^{\circ} \mathrm{C}$.

A socket (17) is designated for installation of a Colutron ion beam viewing system to allow the determination and adjustment of beam profiles of the low energy ion beams insitu. It consists of a set of stacked beam attenuation grids, a luminescent screen and a channel plate for light amplification. The screen emits light under ion bombardment with the local intensity being proportional to the local current density of the beam. The light emission is amplified by the channel plate and observed by a image intensified CCD camera with telescope optics from a flange at the chamber rear side opposite to the high energy beam entrance. 


\section{Operation of the setup}

\subsection{Properties of the ion beams}

First investigations using the new dual beam facility are focused on sputtering of tungsten by simultaneous impact of $\mathrm{D}$ and $\mathrm{C}$ and on sputtering of $\mathrm{C}$ by simultaneous impact of $\mathrm{W}$ and $\mathrm{D}$. Therefore, the test results discussed in the following were obtained from operating the duoplasmatron source with deuterium and by operating the sputter source with $\mathrm{C}$ and $\mathrm{W}$ cathode targets respectively.

The mass-spectrum of the primary ion beam extracted from the duoplasmatron system was obtained by measuring the beam current at the target during a linear sweep of the mass separation magnet current. Figure 3 a shows the resulting spectrum for source operation with deuterium working gas at an accelerating voltage of $9 \mathrm{kV}$. The source produces deuterium ions as well as molecules. The base pressure of the duoplasmatron is $6 \times 10^{-8}$ mbar and consequently the fractions of $\mathrm{H}$ molecule fractions and carbon and oxygen molecule fractions are small compared to the $\mathrm{D}_{3}^{+}$fractions. To obtain the particle current of a given molecule in the mass spectrum, the measured electrical current has to be multiplied by the number of atoms per respective molecule. The $\mathrm{D}_{3}^{+}$molecule dominates the total D-particle current with a fraction of $\approx 80 \%$ and is therefore used for bombardment to achieve the highest possible fluence. Another advantage is that the energy per ion is only a third of the energy gain by a given acceleration voltage, which correspondingly lowers the minimum energy of ions the system can deliver. Mass-separation of the primary beam ensures that only the selected $\mathrm{D}_{3}^{+}$species reaches the target, while impurities, which could greatly affect sputtering measurements, are entirely suppressed because no other molecule species with mass 6 exists.

The Cesium ion-beam system operated with a carbon sputter target is generating a 


\section{Page 11}

variety of different carbon molecule radicals (Figure 3b). The intensity of the beam decreases with the amount of carbon nuclei per molecule radical. The availability of the different radicals is useful to increase the $\mathrm{C}$ fluence and/or to decrease the energy per nuclei. In case of tungsten sputter target, the system cannot separate isotopes and every peak in the mass-spectrum contains all $\mathrm{W}$ isotopes (Figure 3c). The low mass resolution of the magnet is in this case beneficial as it effectively increases the intensity of the $\mathrm{W}$ beam, which has a low total current due to the lower sputtering rate of the cathode. The different peaks correspond to a different amount of oxygen and carbon nuclei per molecule radical [16]. Additionally, two small peaks of ions, containing two tungsten nuclei can be observed. Ions of fusion relevant elements, which can be obtained using the source and their expected fluxes, are summarised in Table 1. For carbon and tungsten, the listed values were directly measured. Where experimental values were not yet available, respective values were taken from [16] and scaled using the ratio of the measured $\mathrm{C}$ and $\mathrm{W}$ currents to their respective values in [16].

The current-voltage characteristic of the $\mathrm{D}$ and $\mathrm{C}$ ion fluxes as function of particle energy is shown in Figure 4. The right scale of the graph shows the fluence that can be achieved after 8 hours, i.e. one working day. From this graph the ratio of carbon to deuterium flux can be estimated as a function of the energies of the corresponding ions. Thus, using a $9 \mathrm{keV} \mathrm{D}_{3}^{+}$ion beam the achievable fluence is $1.4 \times 10^{24} \mathrm{D} / \mathrm{m}^{2}$, which is sufficient for studying the effects connected with sputtering of tungsten and D retention [17]. To decrease the beam current at a given acceleration voltage the beam can be defocused by the Einzel lens.

To achieve a homogeneous profile of the solid-state ion beam over the complete image of the beam defining aperture (Figure 5a, C irradiation on W), it is necessary to shift the beam focus away from the surface of the bombarded sample, which however leads to a 
current decrease by a factor of $\approx 3$. With the focus at the target surface one obtains a hollow beam profile as image of the beam cross-section at the sputter cathode (Figure $5 \mathrm{~b}, \mathrm{C}$ irradiation of W). In contrast to the sputter source beam, the profile of the gaseous ion beam is continuous at any focusing potential (Figure 5c, D irradiation of a-C:H film), because in this case the magnet has the same focal length both in the vertical and horizontal beam plane. Both systems provide highly reproducible beam characteristics at given operating parameters like discharge currents, extraction and focusing voltages etc. However, the presently used approach to quantify the beam profile by optical analysis of irradiated target spots is not flexible and fast enough for easy modification of irradiation parameters. To allow in-situ beam profile measurement for quick adjustment of beam shape and overlap, the beam viewing system described in section 2.4 will be installed for future experiments.

Irradiation of a-C:H films on Si wafers has been used also to verify the overlap of the low energy and high energy beams. The divergence of the beams shown in Figure 6a was observed in first irradiations after assembly. This was a result of a slight mismatch between target surface and the chamber centre where the axes of the two beam lines intersect. The final adjustment was performed by appropriate shift of the target surface in the direction normal to the surface. Figure $6 \mathrm{~b}$ shows the finally achieved convergence of the low energy beams and the position of the high energy analysing beam within the irradiated area. In this case, the central spot corresponding to the position of the analysing beam has been obtained by irradiation with a low-energy deuterium beam through the $1 \mathrm{~mm}$ aperture. The target was rotated to obtain a symmetric incidence angle of $15^{\circ}$ for both low energy ion beams during simultaneous irradiation. Possible inhomogeneities at the boundary of the irradiation spots or at the boundary of their overlap zone have no direct influence on the experimental results because in contrast to weight measurements or thermodesorption 
experiments where the measurements reflect an integral over the irradiation zone, ion beam analysis yields local information. Consequently, the only effect of such inhomogeneities is a possible error in the derivation of the local irradiation flux, which, however, can be taken into consideration in the calculation of the local current density from the measured total current. Therefore, the inhomogeneities have no influence on the conclusions based on ion beam measurements.

\subsection{Measurement of the partial fluences}

During the experiment, the currents of each beam are adjusted manually to achieve the desired ratio of solid-state atoms and deuterium ions in the incident ion flux. Continuous adjustments are required because the solid-state ion beam current is varying due to the principle of operation of cesium sputter source [15]. The main parameters used for adjustment are the voltages of the Einzel lenses of the ion-beam systems. During the experiment the desired current ratio is approximately maintained; the exact concentration of every species in the flux is, however, determined after irradiation. Compared to the long irradiation time, short time scatter is generally acceptable and therefore the constant average can be used in numerical simulations with codes like TRIDYN.

As the picoamperemeter used for the current measurements measures the sum of both currents with a sampling interval of several seconds, the beamline shutter (2) is used for short (also about 3-5 seconds) interruptions of the deuterium ion beam in intervals of several minutes to measure the current of the negative ion beam alone. In the resulting data set of beam current vs. time the deuterium beam interruptions appear as short negative spikes (Figure 7a).

A code has been developed to separate the negative and positive data points assuming that the positive data correspond to the sum of both D and solid state ion currents and the negative data correspond to the solid state ion current alone. In the periods of D 
beam interruption the $\mathrm{D}$ beam current is approximated by interpolation (Figure $7 \mathrm{~b}$ ). Then the fraction of solid state ions in the flux is calculated taking into account the number of nuclei per molecule ion and the area of the irradiated spot (Figure 7c). Here it is assumed again that the negative current can be linearly interpolated between the measurements.

\subsection{Measurement of the surface temperature}

For the measurement of the surface temperature a two-wavelength pyrometer is used, which is adjusted onto the beam target area at point (5) (Figure 8). Temperature measurement by scanning of the bombarded surface is difficult due to the complicated dependency of the tungsten emissivity from the concentration of implanted carbon on top during the experiment. To quantify the discrepancies in the temperature measurements of sample surface and sample holder (2), a thermocouple was attached between sample and holder for test measurements. A customised sample was used, with only half of the surface coated by tungsten (1) and the other half left clean (3). As sample substrate polished pyrolitic graphite was used with an intermediate copper layer for the tungsten coating to prevent tungsten film and carbon substrate from chemical interaction and mutual diffusion. The readings of the thermocouple were compared with the corresponding readings of the pyrometer, aimed at points (4) and (5) in (Figure 8). The observed discrepancy of the readings was generally less than $10 \mathrm{~K}$. Temperature measurements obtained by scanning of the surface of tungsten film required re-calibration after each step of the sample heating. Thus, the accuracy of measurement of the temperature of the sample surface is independent on the emissivity of the irradiated surface.

\section{Structure of the surface and in-situ analysis}

Rutherford backscattering (RBS) allows to obtain information on the total concentration of elements, as well as the depth distribution of atomic species in a sample. 
This provides essential data, which could not be obtained in previous experiments, where only the weight change of samples could be measured in-situ. Rutherford backscattering is, however, limited by the depth range of the analysis ions. It is also not very well suited for detection of light impurities in a matrix of heavy elements. This disadvantage can be avoided by using Nuclear reaction analysis (NRA), which is suitable particularly for detection of light elements as deuterium, beryllium, carbon, oxygen and boron isotopes. In cases where samples contain small amounts of metals distributed over a large depth range, RBS analysis is difficult. In this case, proton induced x-ray emission analysis (PIXE) can be used to obtain accurate measurements of the total amount of a given element, however, without depth resolution.

\subsection{Thin films}

To derive sputter yields of a given target material, RBS is used to determine the number of eroded atoms per incident ion. The number of eroded atoms per unit area is obtained by measuring the area density of the studied target material before and after irradiation with a given incident ion fluence. To provide a defined target material layer for the RBS measurements one can either implant a depth marker of another element into the virgin sample or prepare a layer of the target material on a different substrate material. The

suitability of this experimental approach has already been demonstrated [17]. Its advantages are:

i) Accurate depth profiling of the elements involved in ion-surface interactions, projectiles and irradiated materials;

ii) High accuracy measurements of the total concentration of the respective elements;

iii) Investigation of ion-surface interactions at low fluences, which are important for bombardment with heavy elements;

iv) Measurement of the depth distribution of elements depending on fluence, since 
intermediate RBS measurements during irradiation take usually only a few minutes;

v) Surface roughness can be defined by the selected substrate, which is important for materials which structures not allowing high-quality polishing;

vi) Provides detailed experimental data for benchmarking results of simulation codes such as the TRIDYN program [18];

The selection of the optimal layer thickness is affected by several factors. Apart from D diffusion and retention, the penetration depth of projectiles below the surface in the model experiments is typically several tens of nanometers. Combining these scale lengths with the expected amount of eroded material, one obtains a lower limit for the layer thickness. The erosion of irradiated material can be detected as a decrease of the concentration of the layer materials, therefore the layers have to be accessible for IBA down to the substrate material. This restriction is further tightened if an intermediate layer (e.g. copper) is necessary as barrier for deuterium diffusion from the investigated layer into the substrate [17]. Taking into account all boundary conditions one obtains an optimal thickness in the range of $0.2-0.5 \mu \mathrm{m}$ for films of high-Z elements. Films of this thickness allow measurement of the depth profiles of both low- $Z$ and high- $Z$ element simultaneously by RBS as well as depth profiling of $\mathrm{D}$ and $\mathrm{C}$ by NRA. For low-Z elements the optimal thickness can be chosen higher $(\approx 1 \mu \mathrm{m})$ depending on the requirements of the experiment.

\subsection{Application of RBS analysis}

The first experiments in the DBE setup have been performed with $\mathrm{W}$ thin films deposited onto a substrate of polished pyrolitic graphite with an intermediate layer of $\mathrm{Cu}$. The samples were irradiated simultaneously with $9 \mathrm{keV} \mathrm{D}_{3}^{+}$and $12 \mathrm{keV} \mathrm{C}_{2}^{+}$ions with a $\mathrm{C}$ fraction of $11.4 \%$ of the total ion flux and an angle of incidence of $15^{\circ}$ to the surface 


\section{Page 17}

normal for both species. The thickness of the W layer was measured between subsequent irradiation steps by RBS at a scattering angle of $165^{\circ}$ and ${ }^{4} \mathrm{He}$ ions with an energy of $2 \mathrm{MeV}$ (Figure 9a). The constant height of the $\mathrm{W}$ partial spectrum in subsequent measurements demonstrates the accuracy of the beam charge measurement with an error $<3 \%$. The $11.4 \%$ carbon fraction in the $\mathrm{D}+\mathrm{C}$ beam results in stationary sputtering of the $\mathrm{W}$ film and formation of a steady-state carbon profile within the implantation range in the tungsten layer.

The low energy edge of the W partial spectrum corresponds to the interface between $\mathrm{W}$ and $\mathrm{Cu}$ layers. Initially, its shape is determined by the implantation of $\mathrm{W}$ atoms into the $\mathrm{Cu}$ film during the magnetron deposition of the $\mathrm{W}$ layer resulting in a broadening of the transition between the layers. A variation of the lower edge of the $\mathrm{W}$ partial spectrum would indicate laterally non-uniform bombardment with $\mathrm{C}$ and $\mathrm{D}$ beams and correspondingly non-uniform sputtering of the W film. Similar modifications could also be caused by formation of blisters at the $\mathrm{W}-\mathrm{Cu}$ interface as observed in [17], due to the internal stress of the film by accumulation of deuterium. From the absence of such artefacts in the spectra discussed here, one concludes that the beam profile is homogeneous on the scale of the analysis beam diameter $(1 \mathrm{~mm})$.

Figure $9 \mathrm{~b}$ shows the high energy edge of the $\mathrm{W}$ peak corresponding to the surface and the implantation zone. The carbon profile in the implantation zone should stay constant with increasing fluence after the equilibrium between sputtering and implantation is reached. Indeed the magnified view in Figure $9 \mathrm{~b}$ shows that the shape of the high energy edge of the $\mathrm{W}$ partial spectrum remains constant within the statistical error after an irradiation fluence of $2 \times 10^{18} \mathrm{at} / \mathrm{cm}^{2}$.

Figure 9c shows numerical simulations of the RBS spectra using the SIMNRA program [19]. To accurately model the implantation zone, stacked layers with varying 
$\mathrm{C} / \mathrm{W}$ concentration ratios were used to describe the target structure. The simulated spectra were fitted to the experimental data by varying the layers thickness and composition. By integration over the resulting $\mathrm{W}$ depth profile one obtains finally the total remaining $\mathrm{W}$ area density in the bombarded film. The numerical analysis of the RBS spectra shows that the film thickness decreases by $\approx 100 \times 10^{15} \mathrm{at} / \mathrm{cm}^{2}$ after each irradiation step with a fluence of $\approx 2 \times 10^{18} \mathrm{at} / \mathrm{cm}^{2}$, corresponding to a stationary erosion yield of $\approx 5 \%$. A deviation of $\approx 10 \times 10^{15} \mathrm{~W} / \mathrm{cm}^{2}$ from the best fit is already well visible and represents the upper limit for the error in the measurement of the film thickness. The error originating from uncertainties in the stopping power cancels out because the erosion is derived from the difference of two measurements. This approach is valid as long as the amount of sputtered material in subsequent irradiations is much less than the remaining layer thickness.

\subsection{Application of NRA}

NRA measurements can be used to determine the total amount of implanted D and C using a ${ }^{3} \mathrm{He}$ analysis beam and detecting protons created in the nuclear reactions $\mathrm{D}\left({ }^{3} \mathrm{He}, \mathrm{p}\right) \alpha$ and ${ }^{12} \mathrm{C}\left({ }^{3} \mathrm{He}, \mathrm{p}\right){ }^{14} \mathrm{~N}$. Additionally, in the case of deuterium, depth profiles can be determined from the energy spectrum of the created $\alpha$-particles. In the case of carbon, where the depth profile has to be measured by RBS, the total area density derived from the integral over the depth profile has a quite limited accuracy of $\approx 20 \%$. By NRA, the total amount of implanted $\mathrm{C}$ follows directly from the corresponding proton-peaks, which reduces the statistical error to the error of the beam charge integration.

The proton counter used in the DBE setup has a solid angle of $61.635 \mathrm{msr}$ and is shielded by a $12 \mu \mathrm{m}$ Mylar foil to prevent backscattered ${ }^{3} \mathrm{He}$ from dominating the detected particle spectrum with resulting detector pile up and excessive dead-time. Due to the large solid angle of the detector, accurate NRA measurements can be obtained already with 
analysis beam charges of $\approx 1 \mu \mathrm{C}$. At these beam charges D-depletion by the measurement process itself is negligible $\left(\approx 10^{-3}\right.$ for tungsten with implanted $\mathrm{D}$ and $\left.\mathrm{C}[20]\right)$ and consequently the analysis beam has no detectable influence on target integrity. However, for measurements of $\mathrm{D}$ depth profiles, where higher beam charges up to $50 \mu \mathrm{C}$ are required to obtain a sufficiently small counting statistical error, D-depletion by the measurement has to be taken into account. This is done by splitting a measurement in several subsequent steps. At each step, both the $\alpha$-spectrum and the p-spectrum are recorded. A possible Ddepletion leads to a corresponding decrease of the p-count integral. For the determination of the D-depth profile, the $\alpha$-particle spectra are all summed up with the D-depletion taken into account by a correction factor derived from the respective p-count integrals.

For the quantitative determination of $\mathrm{D}$ and $\mathrm{C}$ area densities from the recorded $\mathrm{p}$ spectra, calibration samples with hard a-C:D layers deposited on a Si-substrate are used. The $\mathrm{D}$ and $\mathrm{C}$ density in such layers can be accurately determined by combining ellipsometry measurements with RBS and NRA [21]. From the known D- and C-area density in the calibration sample one obtains calibration factors for the respective NRA proton peak integrals. The NRA spectrum of the used calibration sample is shown in Figure 10a. The ${ }^{3} \mathrm{He}$ reaction with carbon leads to 3 peaks in the spectrum because the ${ }^{14} \mathrm{~N}$ produced in the reaction can be created in an excited state with correspondingly less energy left for the proton. The peaks $\mathrm{p} 0, \mathrm{p} 1$ and $\mathrm{p} 2$ correspond to protons from reactions with ${ }^{14} \mathrm{~N}$ in ground state, 2.313 MeV excited state and 3.948 MeV excited state [22]. The broadened peak centered around channel 440 is due to protons from the $\mathrm{D}\left({ }^{3} \mathrm{He}, \mathrm{p}\right) \alpha$ reaction. The broadening in comparison to the carbon-related peaks results from the higher energy of the protons. Because the thickness of the active detector layer is not sufficient to stop the protons entirely, they lose only a fraction of their energy on their path through the detector layer, which leads to a broader distribution of the deposited energy by the corresponding 
energy loss straggling. By integration of the C- and D-related proton peaks and multiplication with the respective calibration factors one obtains directly the corresponding total area density of the respective element.

First results on the formation of $\mathrm{D}$ inventories in tungsten studied by D-irradiation in the DBE setup with in-situ NRA analysis are discussed in [17]. A first application of NRA for detection of carbon was the study of $\mathrm{C}$ implantation by bombardment of tungsten with $12 \mathrm{keV} C_{2}^{+}$ions at normal incidence. As discussed in [23], the sputtering of $\mathrm{W}$ is accompanied by an increase of the concentration of implanted $\mathrm{C}$ finally turning into a continuous growth of a $\mathrm{C}$ layer on top of the $\mathrm{W}$ substrate. However, weight-loss measurements as described in [21], allow no separate quantification of C-implantation and $\mathrm{W}$-erosion because the weight change is entirely dominated by the $\mathrm{W}$-sputtering. In contrast to that, ion beam analysis with ${ }^{3} \mathrm{He}$ allows simultaneous measurement of the Wand C- depth profile by means of RBS as described in subsection 4.2 and accurate measurement of the total D- and C areal density by means of NRA.

The evolution of the proton NRA peak p1 with incident $\mathrm{C}$ fluence is shown in Figure 10b. The small peak around channel 198 correspond to protons created by nuclear reactions with the implanted $\mathrm{C}$ layer. One can clearly see the increase of the amount of implanted carbon with increasing irradiation fluence. The large peak originates from the carbon substrate. Its shift to lower energy and the low-energy tail are caused by the energy loss of the ${ }^{3} \mathrm{He}$ analysis ions with increasing depth and a correspondingly decreasing energy input to the nuclear reactions. The slight increase of the substrate peak at the highest irradiation fluence is a result of the decreasing W-layer thickness by sputtering, which leads to a corresponding decrease of the ${ }^{3} \mathrm{He}$ energy loss. 


\section{Conclusions}

A dual beam ion irradiation facility has been developed for the study of sputtering and surface modifications by multi-species ion bombardment of materials and for the investigation of properties of mixed material surfaces. The key characteristics of the DBE setup is the use of in-situ surface diagnostics, which can be upgraded depending on the experimental requirements. Utilization of ion beam analysis coupled with thin films as samples avoids the low accuracy and lack of species resolution in previously used weightloss measurements. It allows the precise determination of species concentrations and of the incident flux, separate depth profiling of different species in the surface region of the solid material and independent observation of both implantation and sputtering dynamics. The experimental data provide in turn information for detailed validation and benchmarking of Monte-Carlo simulation codes by consistent fits of modelled target-projectile systems with varying irradiation conditions. This will help to improve the understanding of the underlying basic processes and consequently to improve predictive quality of numerical models for the complex dynamics of mixed material systems in plasma technology and fusion.

\section{Acknowledgements}

The authors would like to thank Drs. J. Roth, V. Alimov, K. Ertl, Ch. Linsmeier and M. Mayer for their suggestions and helpful discussions during construction, procurement and assembly of the dual-beam facility. We would also like to thank Dipl.-Ing. B. Plöckl for the engineering support and P. Matern, R. Lang, W. Hohlenburger and R. Hoffmann for their technical support. 


\section{References}

[1] R. Behrisch. Sputtering by particle bombardment I. Topics in applied physics, Vol. 47, Berlin/Heidelberg/New York: Springer-Verlag, (1981).

[2] W. Eckstein Computer Simulation of Ion-Solid Interactions. Springer Series in Material Science, Vol.10 Springer, Berlin, (1991).

[3] J.P. Biersack, Radiation Effects vol.19, p.249 (1973).

[4] K. Schmid, J. Roth. J. of Nucl. Mat. Vol. 302 p.96-103 (2002).

[5] J. Luthin, C. Linsmeier. Surf. Sci. 454-456 p.78-82 (2000).

[6] ITER Physics Base, Nucl. Fus. 39 p.2137-2638 (1999).

[7] G. Janeschitz, ITER JCT and ITER HTs, J. Nucl. Mat. vol. 290-293 p.1 (2001).

[8] D. Meade, et al., Mission and design of the fusion ignition research experiment, in: Proceedings of the 18th IAEA Conf. on Fusion Energy, Sorrento, Italy October, 2000, (CD-ROM), pp. IAEA-CN-77/FTP2/16, IAEA, Vienna, 2001.

[9] S. Nishio, et al., Conceptional design of advanced steady-state tokamak reactor, in: Proceedings of the 18th Conf. on Fusion Energy, Sorrento, Italy October, 2000, (CDROM), pp. IAEA-CN-77/FTP2/14, IAEA, Vienna, 2001.

[10] K. Krieger et al., J. Nucl. Mat. Vol.266 -269 p.207 (1999).

[11] W. Eckstein, K. Krieger, J. Roth, J. Nucl. Mat. Vol.258-263 p.912-916 (1998).

[12] K.Krieger, J.Roth, J. of Nucl. Mat., vol.290-293 p.107-111 (2001).

[13] K.Schmid, J.Roth, J. of Nucl. Mat. vol.313 -316 p.302 -310 (2003). 
[14] A.A. Haasz, J. Davis, Nucl. Instrum. and Methods in Phys. Res., vol.B83, p.117 (1993).

[15] G.D. Alton, Nucl. Instr. and Methods in Phys. Res. B73 p.221-288 (1993).

[16] R. Middleton. A Negative-Ion Cookbook; Department of Physics, University of Pennsylvania, Philadelphia, PA 19104 (1989).

[17] I. Bizyukov, K. Krieger, N. Azarenkov, S. Levchuk, Ch. Linsmeier, J. Nucl. Mat. vol.337-339 p.965 (2005).

[18] W. Moeller, W. Eckstein, J.P. Biersack, Comput. Phys. Commun. Vol.51 p.355 (1988).

[19] M. Mayer, MPI f. Plasmaphysik Technical report IPP 113 (9).

[20] V. Alimov (private communication).

[21] T. Schwarz-Selinger, A. von Keudell, and W. Jacob. J. Appl. Phys., vol. 86, \#7, p.3988, 1 October 1999

[22] S.Y. Tong, W.N. Lennard, P.F.A. Alkemada, I.V. Mitchell, Nucl. Instrum. Methods vol. B45, p.41, (1990)

[23] W. Eckstein, J. Roth, Nucl. Instrum. Methods in Phys. Res. vol. B53, p.279, (1991) 
Page 24

\section{Tables}

\begin{tabular}{|c|c|c|c|c|c|}
\hline & $2 \times 10^{17}$ & $\mathrm{TiH}$ & $10^{17}$ & $\mathrm{TaH}$ & $2 \times 10^{15}$ \\
\hline $\mathrm{BeH}$ & $2 \times 10^{16}$ & $\mathrm{VH}$ & $10^{16}$ & $\mathrm{TaC}$ & $5 \times 10^{16}$ \\
\hline $\mathrm{BeO}$ & $10^{17}$ & $\mathrm{VC}$ & $3 \times 10^{16}$ & $\mathrm{TaO}_{2}$ & $1.5 \times 10^{17}$ \\
\hline $\mathrm{C}$ & $10^{18}$ & $\mathrm{Si}$ & $10^{18}$ & $\mathrm{~W}$ & $10^{16}$ \\
\hline \multirow[t]{2}{*}{$\mathrm{O}$} & $10^{18}$ & $\mathrm{Fe}$ & $6 \times 10^{16}$ & $\mathrm{WC}$ & $2 \times 10^{16}$ \\
\hline & & $\mathrm{Ni}$ & $5 \times 10^{17}$ & $\mathrm{WO}_{3}$ & $2 \times 10^{16}$ \\
\hline
\end{tabular}

Table 1. Expected fluxes $\left(\mathrm{m}^{-2} \mathrm{~s}^{-1}\right)$ of some negative ions of fusion relevant elements. 


\section{List of figure captions}

Figure 1. Schematic view of the Dual Beam Experiment setup.

Figure 2. Design of the flange installed on top of the target vacuum chamber. 1, 3- beam aperture systems, 2, 4 - beam shutters, 5 - Faraday cup, $6-$ target holder, $7-165^{\circ}$ RBS detector, $8-135^{\circ}$ RBS detector, $9-105^{\circ}$ RBS detector, 10 - NRA proton counter, 11 PIXE X-ray detector, 12 - detector shutter, 13 - positions for calibration targets, 14, 15 exposure positions, 16 - target heaters, 17 - socket for the screen of the beam viewing system.

Figure 3. (a) Mass spectrum of the primary beam extracted from the Duoplasmatron with deuterium as working gas. (b) Mass spectrum of the primary beam extracted from the Cesium sputter ion source with carbon as cathode material. (c) Mass spectrum of the primary beam extracted from the Cesium sputter ion source with tungsten as cathode material. Here $\mathrm{WX}_{\mathrm{n}}$ and $\mathrm{W}_{2} \mathrm{X}$ refer to various tungsten carbide and oxide molecule ions.

Figure 4. Dependence of $\mathrm{D}$ and $\mathrm{C}$ fluxes on the energy per particle for $\mathrm{D}_{3}$ and $\mathrm{C}_{1}$ ion beams. The right scale represents the fluence that can be achieved during one working day (8 hours).

Figure 5. Profiles of ion beams: (a) irradiation imprint of intentionally defocused C ion beam from the sputter source, (b) image of $\mathrm{C}$ ion beam from the sputter source focused to maximum current, (c) irradiation imprint of D ion beam from the Duoplasmatron source.

Figure 6. Irradiation imprint of misaligned (a) and convergent (b) low energy ion beams. The spot in the centre of (b) denotes the area analysed with the high energy beam. 
Figure 7. Separation of current readings and calculation of the carbon concentration in the total flux: (a) - original current readings; (b) - separated time traces of carbon, deuterium and total currents; (c) - calculated time dependence of the carbon concentration in the total flux.

Figure 8. Target holder with heater 1 - polished pyrolitic graphite with a tungsten magnetron deposited film and copper intermediate layer; 2 - target holding plate; 3 polished carbon area; 4 - position of thermoelement; 5 - position of pyrometer measurement spot.

Figure 9. (a) Decrease of the width of the W partial spectrum with increasing applied fluence. (b) Evolution of the high energy edge of the W partial spectrum during irradiation due to carbon implantation. (c) Fit of the experimental RBS W/implanted C partial spectrum with the SIMNRA program.

Figure 10. Proton spectra (a) Reference sample of a-C:D layer on Si substrate with $1170 \times 10^{15} \mathrm{C} / \mathrm{cm}^{2}$ and $450 \times 10^{15} \mathrm{D} / \mathrm{cm}^{2}$. (b) Increase of $\mathrm{C}$ proton peak by $\mathrm{C}$ implantation and layer growth on top of $\mathrm{W}$ surface due to bombardment with $6 \mathrm{keV} \mathrm{C}$ ions. 


\section{Sputter ion \\ source}

Turbo-molecular pump

30-degree mass analysis magnet

Bombardino vacuum chamber

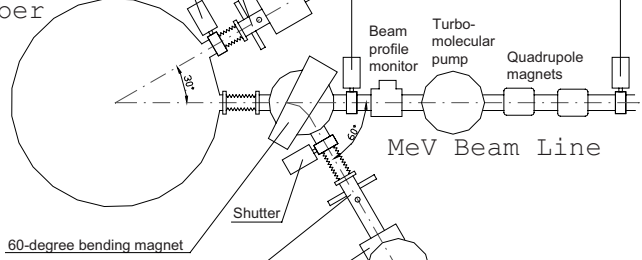

Steerer plate assembly

Einzel lens

Turbo-molecular pump

Duoplasmatron ion source 


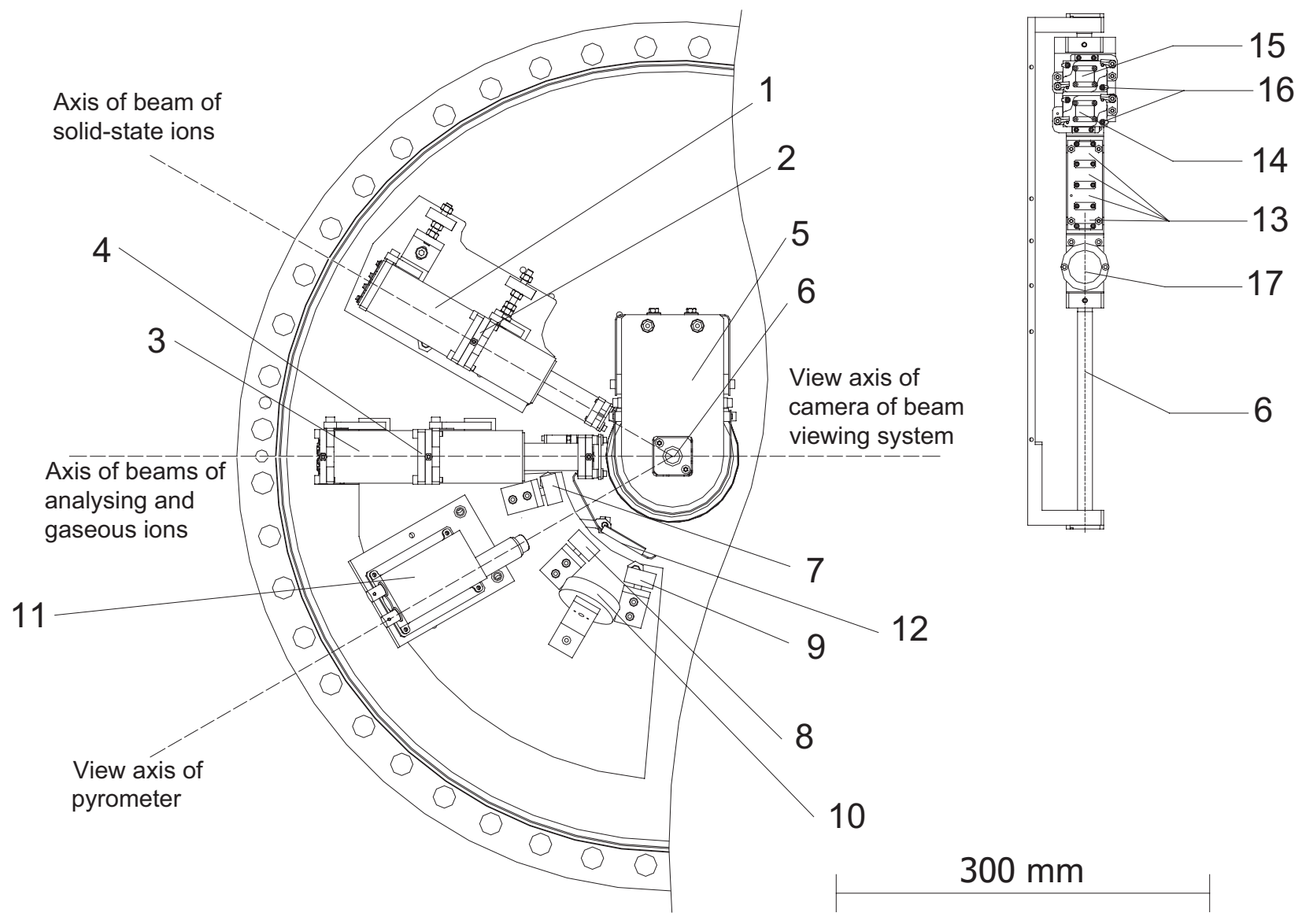




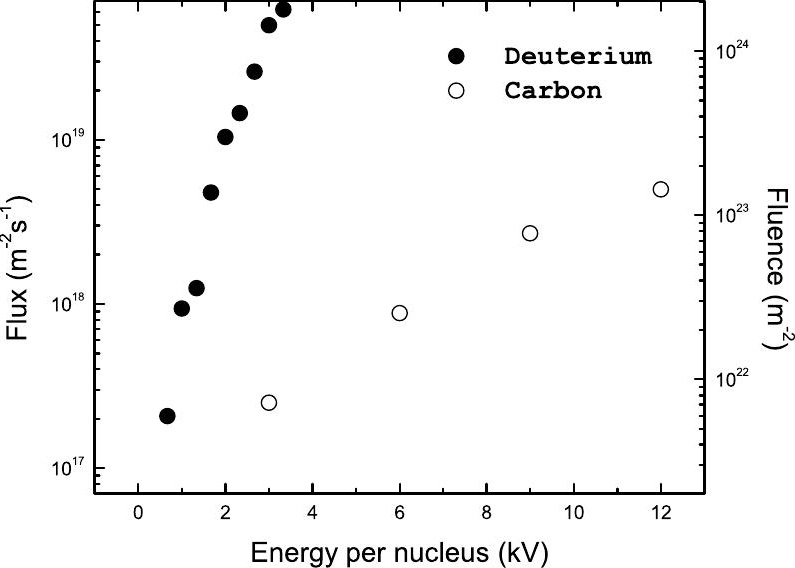



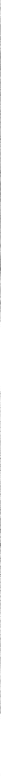

$$
\text { (b) }
$$

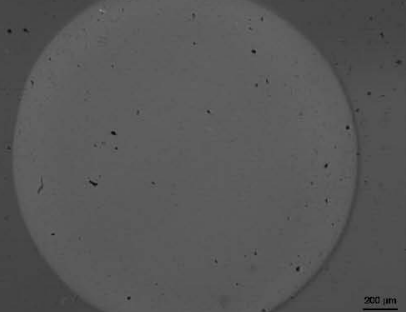

$$
\text { (c) }
$$


$1 \mathrm{mat}$

\section{(a)}

1 茼

(b) 


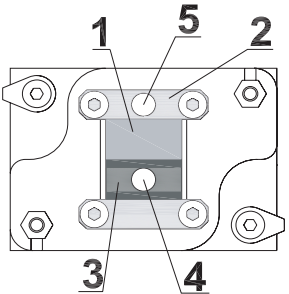




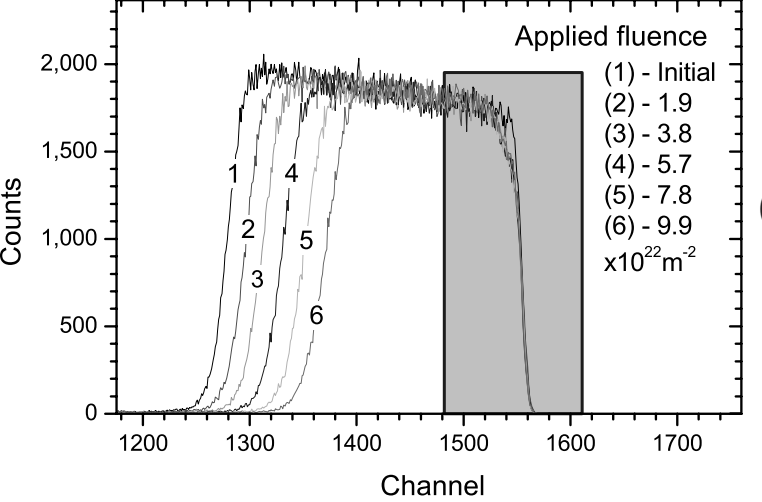

(a)

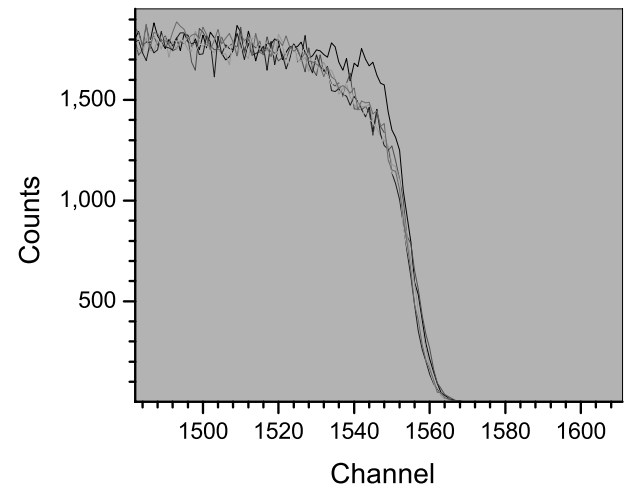

(b)

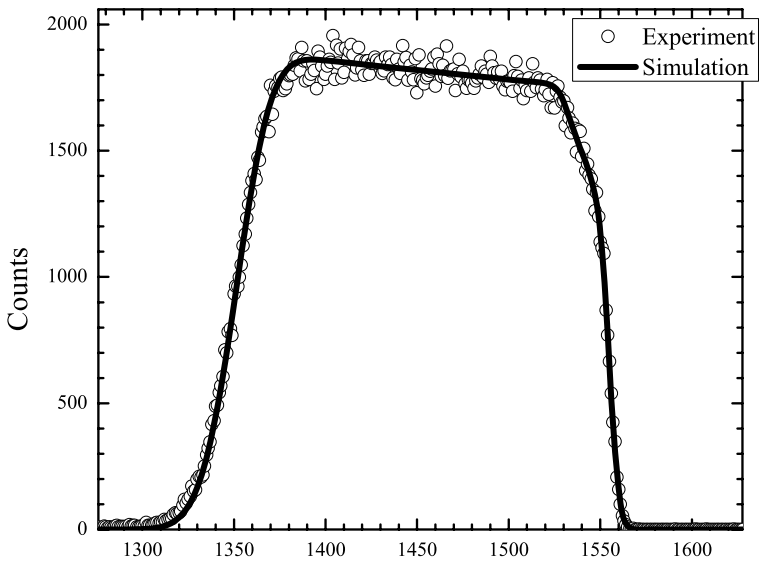

(c) Channel 
